\title{
iScience
}

\section{Article \\ Nature-inspired robust hydrochromic film for dual anticounterfeiting}

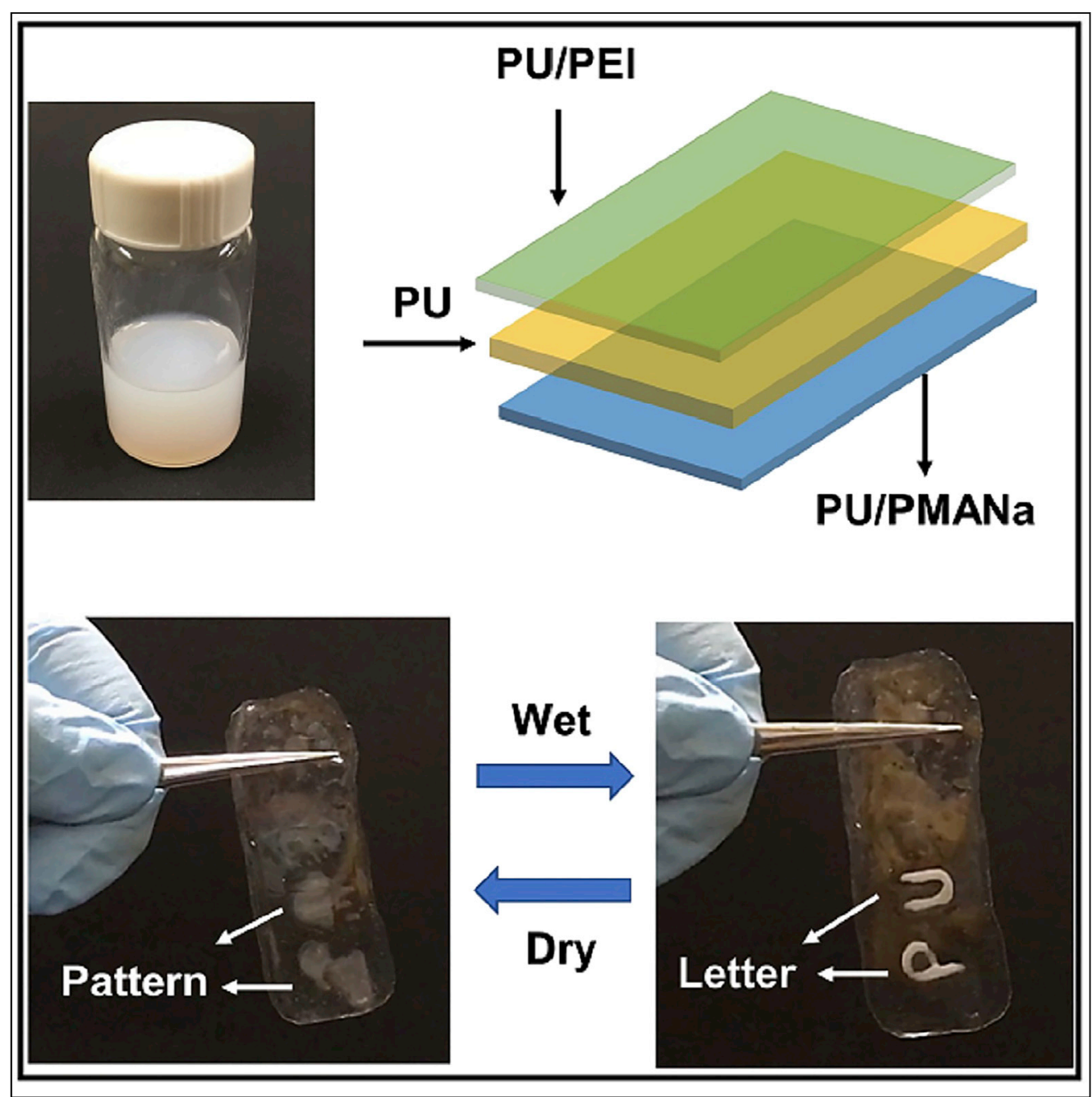

Pengxiang Si,

Mingrui Liang,

Manyou Sun,

Boxin Zhao

zhaob@uwaterloo.ca

Highlights

Micro-structures are

formed by self-assembly

of polyurethane-

polyelectrolyte colloids

Information changes from

one pattern to another

within $3 \mathrm{~s}$ when exposed

to water

The hydrochromic films are mechanically robust in

both dry and wet state 


\section{Article \\ Nature-inspired robust hydrochromic film for dual anticounterfeiting}

\author{
Pengxiang Si, ${ }^{1}$ Mingrui Liang, ${ }^{1}$ Manyou Sun, ${ }^{1}$ and Boxin Zhao ${ }^{1,2, *}$
}

\section{SUMMARY}

Nature-inspired materials have been actively developed for anticounterfeiting applications. Among a variety of stimuli-responsive anticounterfeiting strategies, hydrochromic materials exhibit reversible color change in response to moisture or water and have the advantage of being easy to authenticate. However, the security level of current hydrochromic anticounterfeiting materials is not sufficient for practical applications since they only exhibit a single anticounterfeiting function, where the information switches between visible and invisible. To improve the security level and efficiency of hydrochromic anticounterfeiting materials, here we developed a robust dual hydrochromic material via the self-assembly of polyurethane (PU)-polyelectrolytes colloids with which the desired information can not only switch between visible and invisible but also transform from one pattern to another within $3 \mathrm{~s}$ without the need of any external instruments. The bio-inspiration, material design and demonstrated hydrochromic properties might have profound implications for using colloidal complexes to make advanced anticounterfeiting materials.

\section{INTRODUCTION}

Nature is a bountiful source of inspiration for modulating light-matter interactions (Choi et al., 2020; Liu et al., 2019a; Syurik et al., 2017; Vüllers et al., 2016). Plant- or animal-derived materials containing unique nanostructures or microstructures are able to regulate light scattering, providing a wide range of structural color from whiteness to transparency (Jacucci et al., 2020). Scattering occurs when light propagates in a material with refractive index non-uniformities such as scattering centers or particles in bulk materials (Bohren, 1983). Thus, tuning the refractive index of a material via environmental stimuli enables dynamic color modification (Li and Yin, 2019). Hydrochromic materials exhibit reversible color change through humidity or water and have been widely used in displays, sensors, smart windows, and anticounterfeiting labels (Singh et al., 2017; Yu et al., 2020; Zhao et al., 2020). One example from nature is the skeleton flower (Diphylleia grayi) petals, which consist of numerous air cavities in dry weather, enabling light scattering and resulting in white color (Figure 1A, left). However, on a rainy day, those air gaps are filled with water that has a similar refractive index to its petals, minimizing light scattering and leading to a transparent appearance (Figure 1A, right) (Cai et al., 2018; Liu et al., 2019a; Yong et al., 2015; Yoo et al., 2020). Similarly, the tortoise beetle Charidotella egregia displays red color in dry weather and golden color on rainy days due to the filling of air or water in the nano-porous skin (Vigneron et al., 2007).

These interesting phenomena have inspired many researchers to develop a variety of hydrochromic materials for anticounterfeiting applications (Choi et al., 2020; Yu et al., 2018; Zeng et al., 2017). Compared with thermochromic, electrochromic, and photochromic strategies, hydrochromic anticounterfeiting materials are inexpensive, mass producible with printing technologies, and easy to authenticate by the naked eye without external instruments (Huang et al., 2019a; Huang et al., 2019b; Kang et al., 2018; Liu et al., 2019b; Peng et al., 2018; Wang et al., 2018; Yao et al., 2019). However, the security level of hydrochromic materials is not high enough for real uses because they only exhibit single anticounterfeiting function, with the information switching between visible and invisible by water stimuli (Choi et al., 2020; Singh et al., 2017; Yong et al., 2015; Yoo et al., 2020). To improve the safety of the secret information, Zhao et al. developed a thermal and hydrochromic double encrypted system. It requires a high temperature of $180^{\circ} \mathrm{C}$ enabled by an external instrument (Zhao et al., 2020). Note that organic phosphorescence materials could be used for anticounterfeiting; they had been used to realize multi-dimension/hydrochromic anticounterfeiting (Lei et al., 2020; Xiao et al., 2018).

\footnotetext{
${ }^{1}$ Department of Chemical Engineering, Waterloo Institute for Nanotechnology, Institute for Polymer Research, University of Waterloo, 200 University Avenue West, N2L 3G1, Canada

2Lead contact

*Correspondence: zhaob@uwaterloo.ca https://doi.org/10.1016/j.isci. 2021.102652
} 
A
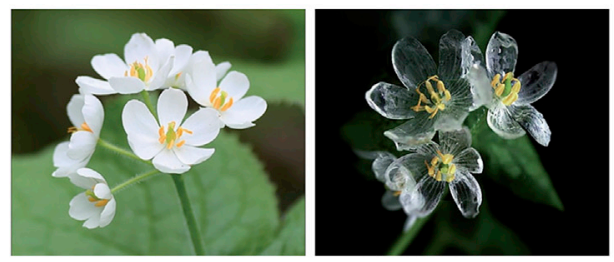

B

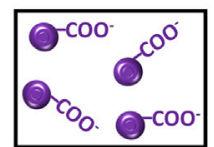

1

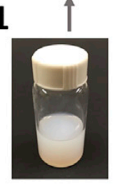

PU water

dispersion c

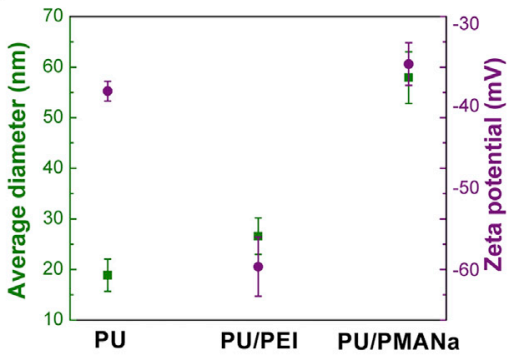

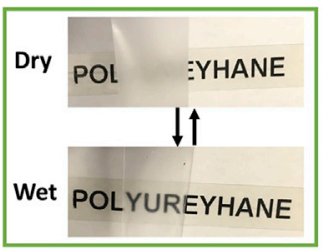

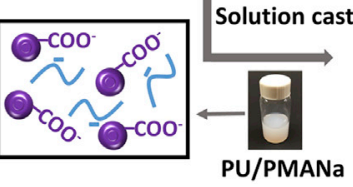

3

PU/PEI film

4

2

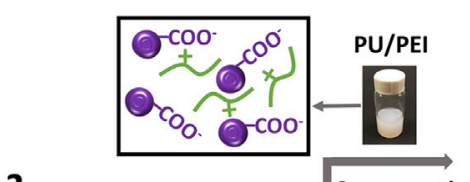

Spray coating
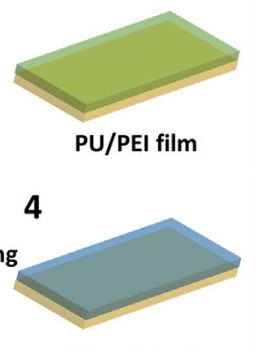

PU/PMANa film

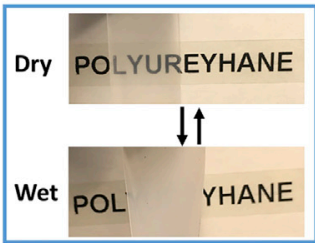

Figure 1. Diphylleia grayi-inspired two types of hydrochromic films are fabricated via polyurethanepolyelectrolyte colloidal dispersion

(A) Photo of the petals of Diphylleia grayi on a dry day (left) and on a rainy day (right) (Yong et al., 2015). Copyright. Royal Society of Chemistry.

(B) Schematic illustration of fabrication of two types of hydrochromic films via PU-polyelectrolyte colloidal dispersion. (1) Photo of anionic PU water dispersion. (2) Fabrication of transparent PU film via naturally drying anionic PU water dispersion on a glass slide at room temperature. (3) Fabrication of PU/PEI hydrochromic film via spray coating PU/PEI colloidal dispersion onto PU film. Photo of PU/PEI hydrochromic film at dry and wet states. (4) Fabrication of PU/PMANa hydrochromic film via solution casting PU/PMANa colloidal dispersion onto PU film. Photo of PU/PMANa hydrochromic film at dry and wet states.

(C) Average diameter and Zeta potential of PU, PU/PEI, and PU/PMANa colloidal dispersions (error bar is the standard deviation; and each sample is measured for 5 times).

Herein, inspired by Diphylleia grayi, we develop a robust dual anticounterfeiting hydrochromic material in which the information can not only switch between visible and invisible but also change from one pattern to another within $3 \mathrm{~s}$ without external instruments, providing opportunities to fabricate more complicated anticounterfeiting patterns for real applications. The designed dual anticounterfeiting hydrochromic material was fabricated using anionic polyurethane (PU) water dispersion and consisted of three layers which respond differently to water stimuli: the top layer changes from opaque to transparent; the middle layer remains transparent; and the bottom layer changes from transparent to opaque. Anionic PU water dispersion was selected as the matrix due to its high transparency, mechanical robustness, and solution processibility. By controlling the deposition and self-assembly of PU colloidal particles during the film formation process, the obtained material exhibits different microscopic structures and transparency.

\section{RESULTS AND DISCUSSION}

\section{Fabrication of hydrochromic films}

The schematic diagram for the preparation of PU/PEI and PU/PMANa hydrochromic films is presented in Figure 1B. The synthesis route of anionic PU water dispersion followed that of our previous work (Si et al., 2020a). Figure $1 \mathrm{~B}_{1}$ displays a photo of the anionic PU water dispersion, which was an amphiphilic polymer containing a hydrophilic carboxyl head from 2,2-bis(hydroxymethyl) propionic acid (DMPA) and hydrophobic core from polypropylene glycol-2000 (PPG-2000) and isophorone diisocyanate (IPDI). The amphiphilic PU in water self-assembled into colloidal particles with an average diameter of $18 \mathrm{~nm}$ and Zeta potential of $-38 \mathrm{mV}$ determined from dynamic light scattering (DLS) data, as seen in Figure 1C. The arrangement of PU colloidal particles during the drying process could significantly affect the optical 
A

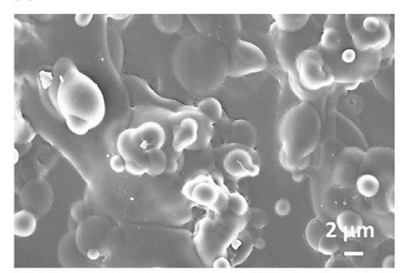

C

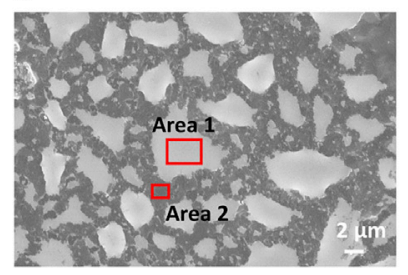

B

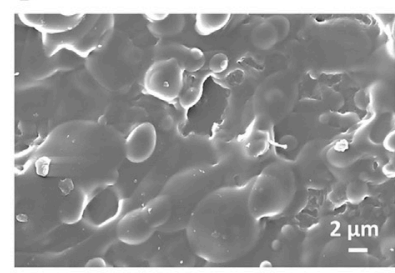

D

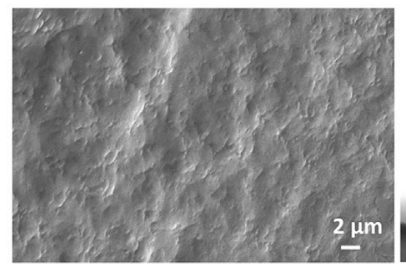

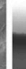

F

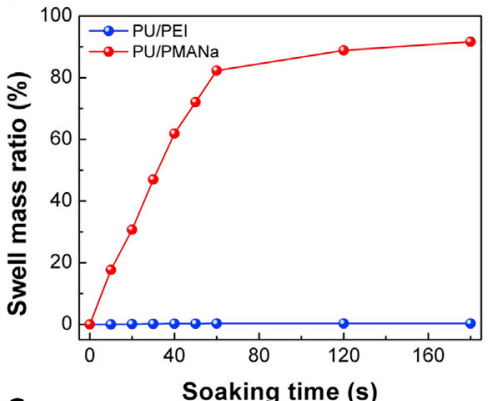

G

PU/PEI $90.5^{\circ} \pm 4.6^{\circ}$ PU/PMANa $73.8^{\circ} \pm 2.8^{\circ}$
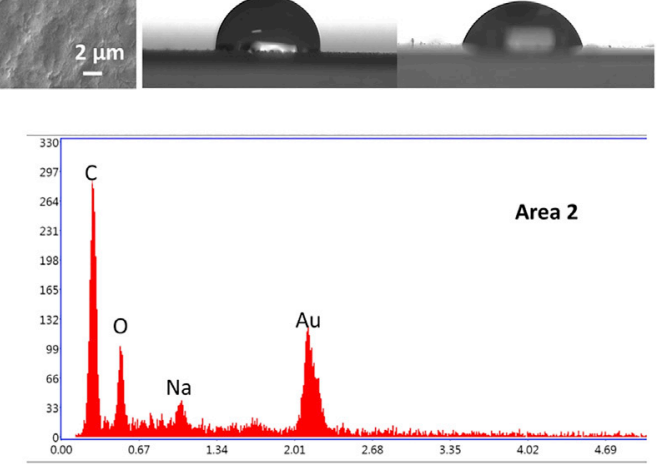

Figure 2. The two types of hydrochromic films have different micro-structures and water swelling behavior

(A) SEM image of PU/PEI hydrochromic film in a dry state.

(B) SEM image of PU/PEI hydrochromic film after soaking in water for $1 \mathrm{~min}$ and drying for $30 \mathrm{~min}$.

(C) Cross-sectional SEM image of PU/PMANa hydrochromic film in a dry state.

(D) Cross-sectional SEM image of PU/PMANa hydrochromic film after soaking in water for 1 min and drying for 30 min.

(E) EDX data of PU/PMANa hydrochromic film.

(F) Water swelling mass ratio of PU/PEI and PU/PMANa hydrochromic films at different soaking times.

(G) Initial water contact angles on PU/PEI (left) and PU/PMANa (right) hydrochromic films, (each sample was measured for 3 times).

transparency of dried films. To fabricate a transparent PU substrate, the anionic PU water dispersion was cast onto a glass slide. As the water slowly evaporated at room temperature, PU colloidal particles diffused and entangled with each other to form a transparent polymer film (Figure $1 \mathrm{~B}_{2}$ ).

Inspired by Diphylleia grayi, the fabrication of a thin layer of micro-particles on a transparent surface could effectively scatter visible light, leading to an opaque appearance (Sun and Bhushan, 2019). Cationic polyelectrolyte PEI was blended into anionic PU water dispersion with the protection of ammonium hydroxide to form a stable PU/PEI dispersion (Si et al., 2020a). During spray coating, the high pressure of the air flow divided PU/PEI dispersion into numerous microdroplets. As the ammonium hydroxide quickly evaporated, PU and PEl aggregated within these microwater droplets to form PU/PEI micro-particles on the PU substrate. Similar to Diphylleia grayi, the obtained PU film coated with the PU/PEI micro-particles was hydrochromic, changing between opaque in the dry state and transparent in the wet state (Figure 1 $\mathrm{B}_{3}$, Video S1). In contrast with PU/PEI film, anionic polyelectrolyte PMANa was mixed with anionic PU water dispersion to produce another type of hydrochromic film that changed from transparent to opaque via water stimuli. The PU/PMANa dispersion was directly cast onto PU substrate. The dried PU/PMANa film was visually transparent in the dry state and opaque in the wet state (Figure $1 \mathrm{~B}_{4}$, Video S2). Both PU/PEI and PU/PMANa dispersions exhibited an average diameter less than $60 \mathrm{~nm}$ and Zeta potential less than $-30 \mathrm{mV}$, indicating good colloidal stability for further processing (Figure 1C).

\section{Morphologies and swelling behavior of hydrochromic films}

To investigate the mechanism of the above two types of hydrochromic films, the morphologies of the PU/ $\mathrm{PEI}$ and PU/PMANa hydrochromic films were examined with a scanning electron microscope (SEM). The 
spray-coated PU/PEl particles had a diameter ranging from $400 \mathrm{~nm}$ to $20 \mu \mathrm{m}$ in their dry state (Figure 2A). After soaking in water for $1 \mathrm{~min}$ and drying for $30 \mathrm{~min}$, the PU/PEI hydrochromic films maintained the same microstructures as in their dry state, indicating that the PU/PEI particles are stable in water (Figure 2B). Compared with the PU/PEI hydrochromic film, the surface of the PU/PMANa hydrochromic film was smooth but had micro-domains inside the material (Figure 2C). These irregular domains were formed via self-assembly of PU and PMANa and had a diameter ranging from $400 \mathrm{~nm}$ to $10 \mu \mathrm{m}$ when dry (Si et al., 2020b). These micro-domains on the cross-sectional surface of the dry PU/PMANa after being soaked in water for $1 \mathrm{~min}$ and dried for $30 \mathrm{~min}$ were found disappeared due to the high water affinity and solubility of PU/PMANa micro-domains (Figure 2D). The difference ratio of $\mathrm{Na}$ element from energy-dispersive $\mathrm{X}$-ray spectroscopy (EDX) data indicated these micro-domains were PMANa dominated and the polymer matrix was PU dominated (Figure 2E). To further verify the capability of water absorbance, the swelling mass ratio and water contact angle of PU/PEI and PU/PMANa hydrochromic films were measured. The swelling mass ratios of PU/PEI and PU/PMANa hydrochromic films were $0.2 \mathrm{wt} \%$ and $90 \mathrm{wt} \%$, respectively (Figure $2 \mathrm{~F}$ ). This could be explained by PEI physically cross-linking with the carboxyl groups on PU through electrostatic attraction to increase the hydrophobicity of PU, whereas the addition of PMANa would introduce more carboxyl groups to increase the hydrophilicity of PU. Therefore, the PU/PEI hydrochromic film had a higher initial water contact angle $\left(90.5^{\circ} \pm 4.6^{\circ}\right)$ than the PU/PMANa hydrochromic film $\left(73.8^{\circ} \pm 2.8^{\circ}\right.$ ) (Figure $2 \mathrm{G}$ ). The water contact angle on the PU/PMANa film decreased within $180 \mathrm{~s}$ due to its hydrophilicity and good water absorbance (Figure S1A), whereas the contact angle of PU/PEI film maintained almost the same (Figure S1B). The significance of testing contact angle is to reveal the difference of wettability and water absorbance rate of PU/PEI and PU/PMANa film. The PU/PMANa absorbs water quickly but PU/PEI does not. Therefore, PU/PMANa turns white but PU/PEI remains transparent. Actually, PU/PEI film can eventually turn white after being soaked in water for $6 \mathrm{hr}$.

\section{Optical transmittance of hydrochromic films}

The optical transmittance of PU/PEI and PU/PMANa hydrochromic films in their dry and wet states was found to be dependent on coating thickness. We prepared two different thicknesses of $5 \mu \mathrm{m}$ and $30 \mu \mathrm{m}$ for each of the PU/PEI and PU/PMANa coating layers, which were measured by a 3D laser microscope (Figure $3 \mathrm{~A}$ ). As a control experiment, the neat PU exhibited high transmittance (above 95\%) in both the dry and wet states without any hydrochromic behavior. The PU/PEI and PU/PMANa films with 5- $\mu$ m coating thickness showed a transmittance change from $60 \%$ to $88 \%$ and $86 \%$ to $25 \%$ for their dry to wet states, respectively. The thicker films showed similar trends but a lower transmittance in both their dry and wet states (Figures $3 \mathrm{~B}$ and $3 \mathrm{C}$ ). The hydrochromic properties of PU/PEI and PU/PMANa films were reversible with no sign of degradation for 20 dry-wet cycles (Figure 3D). The sizes of PU/PEI particles and PU/PMANa micro-domains varied from $400 \mathrm{~nm}$ to $20 \mu \mathrm{m}$, which spanned beyond the wavelength of the visible light $(400 \mathrm{~nm}-800 \mathrm{~nm})$ The large particle size and the phenomenon of thickness-dependent transmittance collectively confirmed the Mie scattering mechanism of PU/PEI and PU/PMANa films (Rybin et al., 2009; Yao et al., 2019).

\section{Mie scattering effect of hydrochromic films}

Figure 4 shows a schematic diagram of the scattering effect in PU/PEI and PU/PMANa hydrochromic films in their dry and wet states. The dried PU/PEI hydrochromic composite film was in a scattering state because the path of incident light was disturbed by the discrete PU/PEI micro-particles adhered on the PU surface. In the wet state, water wetted both PU/PEI particles and PU substrate and water filled in the spaces among the PU/PEI particles, replacing the air; the water wetting minimized the overall scattering effect since the refractive index of water $(n=1.33)$ is closer to $P U(n=1.49)$ than to air $(n=1)$ (Figures $4 A$ and $4 B)$. Therefore, the fabrication of PU/PEI particles on the top of PU surface so as to have the air gaps among them was critical to achieve a hydrochromic effect. In contrast, the PU/PEI film showed high transmittance of $95 \%$ when the fabrication method was solution casting instead of spray coating and no change of transmittance was observed after soaking in water for $1 \mathrm{~min}$.

In contrast to the PU/PEI hydrochromic composite film which transmittance increased in wet state, the transmittance of the PU/PMANa hydrochromic composite film significantly decreased when exposed to water. This is because that the PU/PMANa micro-domains were formed inside the PU matrix due to the self-assembly of colloidal solution. The similarity between the refractive indices of PMANa $(n=1.48)$ and $\mathrm{PU}(\mathrm{n}=1.49)$ minimized the scattering effect to achieve a high transparency in the dry state. When soaked in water, the hydrophilic PU/PMANa particles quickly absorbed water, leading to the spreading of water 
A

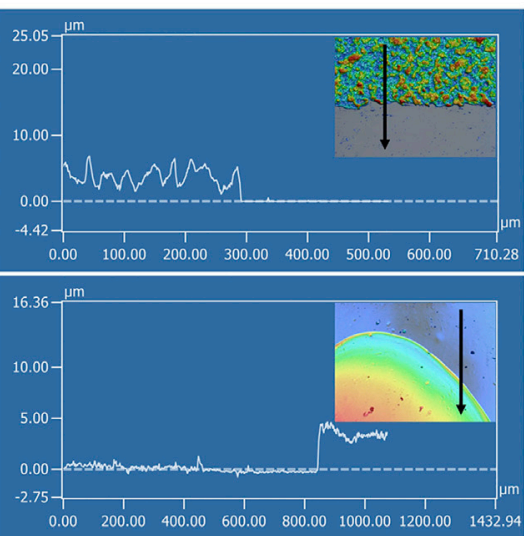

C

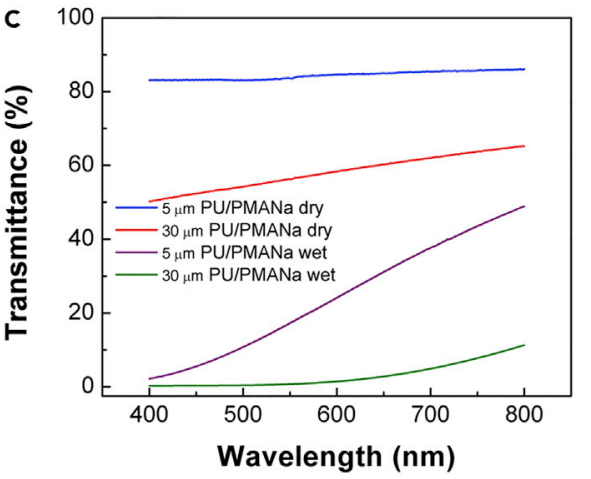

B
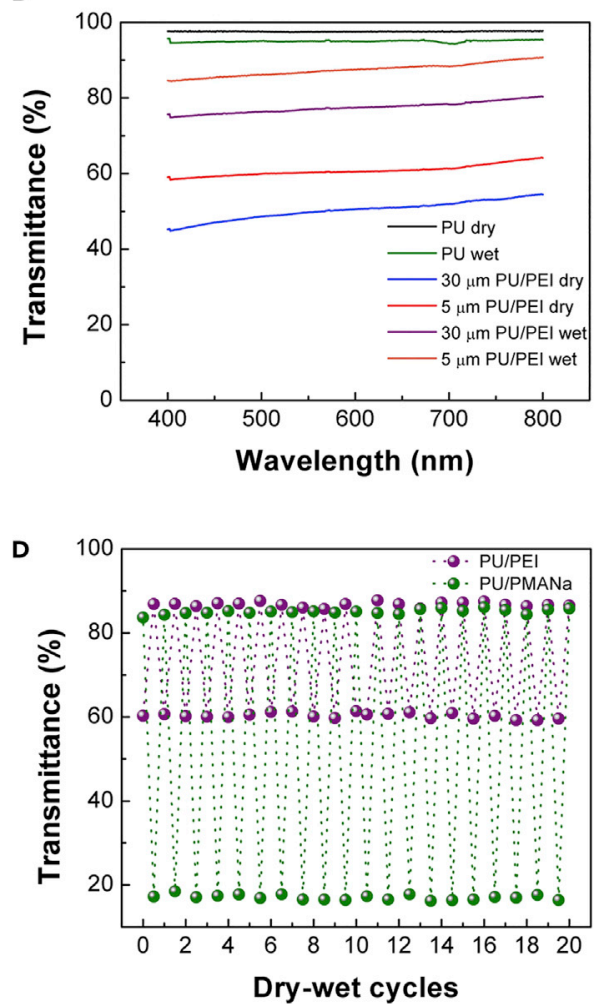

Figure 3. The transmittance of hydrochromic films changes when exposed them in water

(A) Thickness measurements of PU/PEI layer (top) and PU/PMANa layer (bottom) on PU substrate.

(B) Transmittance $(400-800 \mathrm{~nm})$ of PU, PU/PEI hydrochromic film with different thicknesses at dry and wet states.

(C) Transmittance (400-800 nm) of PU/PMANa hydrochromic film with different thicknesses at dry and wet states.

(D) Transmittance $(550 \mathrm{~nm})$ of PU/PEI and PU/PMANa hydrochromic films with $5 \mu \mathrm{m}$ thickness over 20 dry-wet cycles.

inside the film. These PU/PMANa micro-domains acted as scattering centers that decreased the transmittance of the film (Figures 4C and 4D). Notably, it is the water adsorbed in the micro-domains that act as the scattering center for the PU/PMANa composite film. In dry state, the PU/PMANa and PU have similar refractive indices so that the composite film is transparent. In wet state, the absorbed water in the micro-domains has a different refractive index than the PU, subsequently acting as a scattering center while not affecting the film integrity (Figure 2D). These invisible micro-domains once dried could still absorb water. Therefore, the hydrochromic phenomenon of PU/PMANa composite film is reversible.

\section{Mechanical properties of hydrochromic films}

To be more practical for potential applications in anticounterfeiting, the transmittance contrast of hydrochromic films between their dry and wet states needs to be maximized. Therefore, PU/PEI and PU/ PMANa films with $5 \mu \mathrm{m}$ thickness coating layers were selected to fabricate dual anticounterfeiting material. In addition to optical properties, the mechanical properties of anticounterfeiting material are also critical for practical applications. From the tensile stress-strain curves, the PU/PEI hydrochromic film showed a tensile strength of $9.1 \mathrm{MPa}$, breaking elongation of $1600 \%$ and toughness of $84.5 \mathrm{MJ} / \mathrm{m}^{3}$. The PU/PMANa hydrochromic film was not as tough as the PU/PEI hydrochromic film because the non-crosslinkable and intrinsically rigid PMANa blocked the diffusion and entanglement of PU colloidal particles. After soaking in water for $1 \mathrm{~min}$, the tensile strength decreased and breaking elongation increased for both PU/PEI and PU/PMANa hydrochromic films, indicating that the water molecules served as a plasticizer of the polymers. Due to the difference between their water swelling ratios, the PU/PMANa hydrochromic film had a much larger toughness decrease of $20.2 \mathrm{MJ} / \mathrm{m}^{3}$ than the PU/PEI hydrochromic film with $1.1 \mathrm{MJ} / \mathrm{m}^{3}$ (Figure $5 \mathrm{~A}$ ). The adhesive properties of PU/PEI and PU/PMANa hydrochromic films were characterized through a $180^{\circ}$ peeling test to evaluate the bonding strength between the PU/PEI or PU/PMANa coatings and the PU substrate. The test was applied under both dry and wet 
A

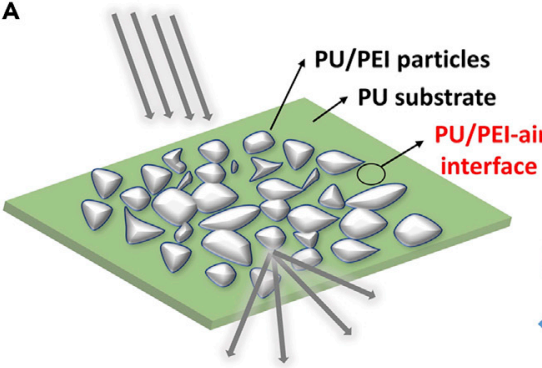

C

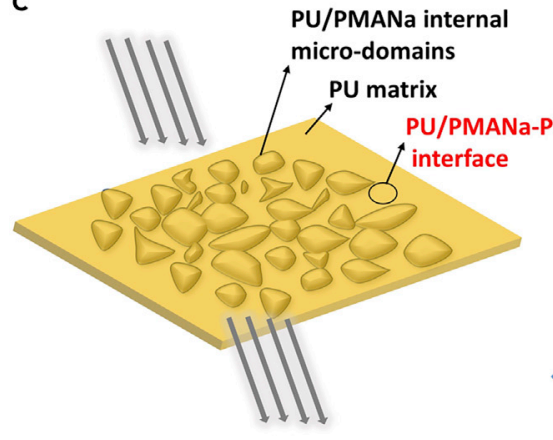

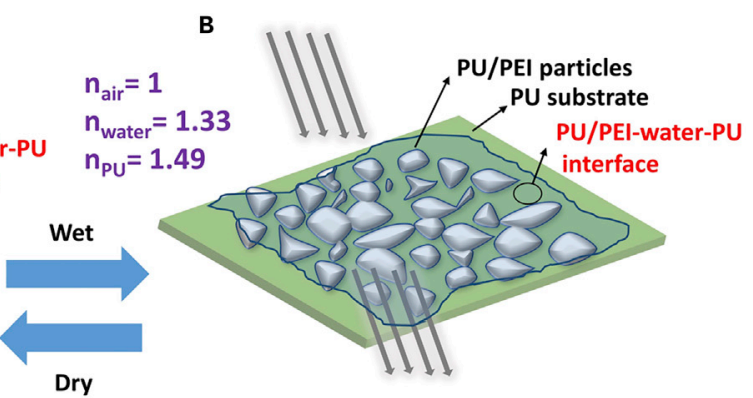

Dry
D

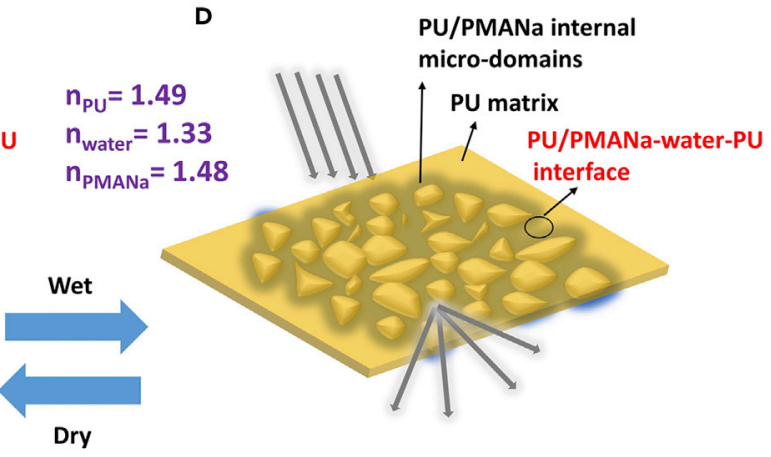

Figure 4. The hydrochromic phenomenon is explained by Mie scattering effect Schematic diagram of the Mie scattering effect of PU/PEI ( $A$ and $B$ ) and PU/PMAMa ( $C$ and D) hydrochromic films in their dry and wet states.

states. After peeling, there was no damage either at the tape/PU/PEI (or tape/PU/PMANa) interface or the PU/PEI/PU (or PU/PMANa/PU) interface, indicating the strong adhesion between the PU/PEI (or PU/PMANa) coatings and the PU substrate. The peel force was higher for the wet state because water softened the polymer and increased its contact area with the tape (Figure 5B). Furthermore, scratch tests were performed to produce PU/PEI and PU/PMANa hydrochromic film surface damage under controlled loading. Figures 5C and 5D exhibited typical scratch track of damages on PU/PEI and PU/PMANa hydrochromic films under an optical microscope with an increasing load from $2 \mathrm{~g}$ to $5000 \mathrm{~g}$ in dry and wet states. The value of critical load was used to determine the maximum load that PU/PEI and PU/PMANa hydrochromic films can tolerate prior to delamination. The delamination of PU/PEI and PU/PMANa films started at about $1000 \mathrm{~g}$ and $500 \mathrm{~g}$, respectively, suggesting good scratch resistance in both dry and wet states. Overall, the toughness, adhesion, durability, and failure resistance of the PU/PEI and PU/PMANa hydrochromic films were robust for practical applications.

\section{Dual anticounterfeiting hydrochromic film}

As a proof of concept, we developed a robust dual anticounterfeiting hydrochromic material by integrating PU/PEI and PU/PMANa coatings on each side of a PU film. The transparent PU substrate of $200 \mu \mathrm{m}$ thickness is the middle layer, the "cloud" pattern made with PU/PEI colloidal dispersion was on the top side, and the "PU" letters made using PU/PMANa colloidal dispersion were on the bottom side of PU film (Figure 6A). The obtained dual anticounterfeiting hydrochromic material exhibited "cloud" patterns in the dry state. After soaked in water for $3 \mathrm{~s}$, the "cloud" patterns changed to "PU" letters (Figure 6B and Video S3). In addition to a PU substrate, we have performed preliminary tests, showing that the dual anticounterfeiting material can also be fabricated on other transparent substrates such as glass, polystyrene, polyethylene terephthalate, etc. Compared to the previous single anticounterfeiting hydrochromic materials on which information can only appear or disappear, our designed material successfully accomplished a complete information change on the same area of material with fast responsive time and without external instruments. More complicated two-dimensional patterns and coded information could be created by a variety of combinations of hydrochromic PU/PEI, PU/PMANa, and non-hydrochromic materials on the same area of materials, providing a dual anticounterfeiting function. 


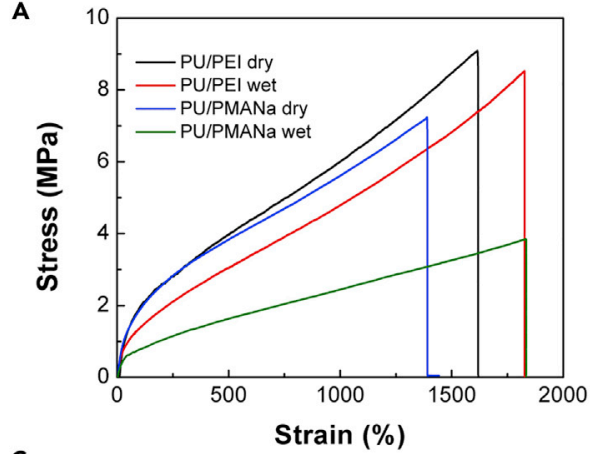

C

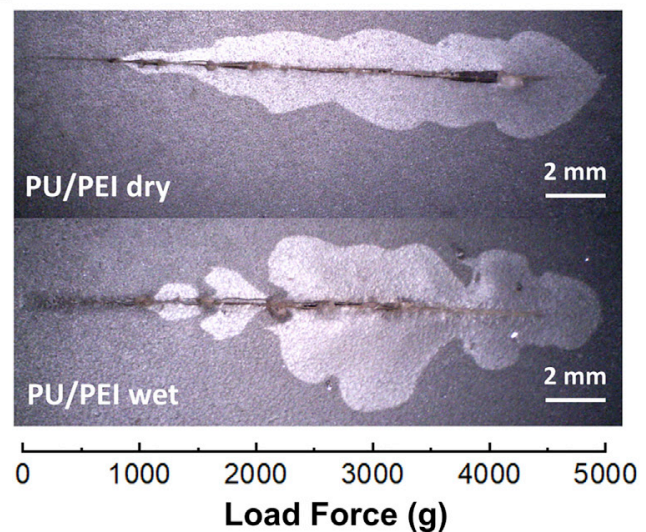

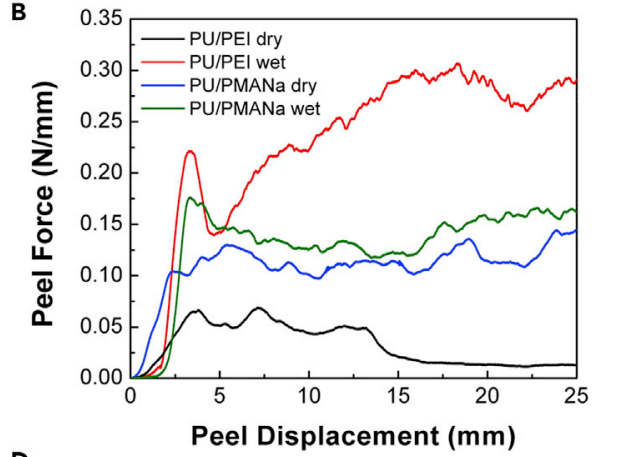

D

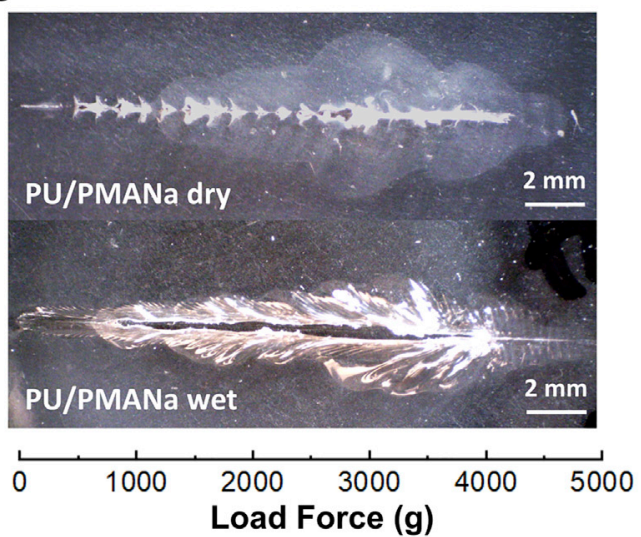

Figure 5. The hydrochromic films are mechanically robust

(A) Stress-strain curves of PU/PEI and PU/PMANa hydrochromic films.

(B-D) (B) Peeling curves of PU/PEI and PU/PMANa hydrochromic films in their dry and wet states. Scratches observed on

$\mathrm{PU} / \mathrm{PEI}(\mathrm{C})$ and PU/PMANa (D) hydrochromic films in dry and wet states by scratch tests.

\section{Conclusion}

In summary, inspired by Diphylleia grayi, we have demonstrated a mechanically robust hydrochromic film based on PU/PEI and PU/PMANa colloidal dispersions for dual anticounterfeiting, wherein the information can not only switch between visible and invisible but also change from one pattern to another within fast responsive time and without external instruments. Water was utilized to tune the refractive index of hydrochromic films in order to modify the light scattering pattern of PU/PEI and PU/PMANa micro-structures, resulting in transmittance change of the material. The water-transparent PU/PEI and water-opaque PU/ PMANa were coated on the top and bottom surfaces of a PU substrate, respectively. After soaked in water, the coated "cloud" patterns made by PU/PEI visually transformed into "PU" letters made by PU/PMANa within $3 \mathrm{~s}$. The designed dual anticounterfeiting hydrochromic films are mechanically robust, quick to respond, inexpensive, environmentally friendly, mass producible, printable, non-destructive, and easy to authenticate with high security. Two-dimensional patterns and codes could be fabricated by a variety of combinations of hydrochromic PU/PEI, PU/PMANa, and non-hydrochromic materials on the same area of materials, showing great potential in fabricating more complicated anticounterfeiting hydrochromic materials in the future.

\section{Limitations of the study}

The transparency contrast between dry and wet states of the PU/PEI layer needs to be further improved in the future. Additionally, the anticounterfeiting material might lose the hydrochromic phenomenon after being continuously soaked in water for longer than one day due to the dissolving of PMANa and hydrolysis of PU.

\section{STAR $\star$ METHODS}

Detailed methods are provided in the online version of this paper and include the following: 


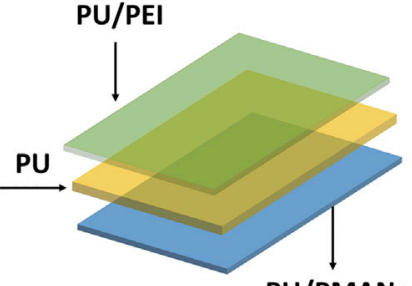

B

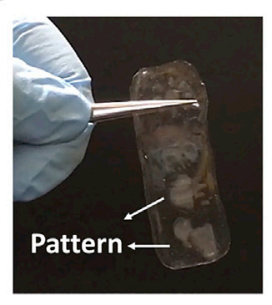

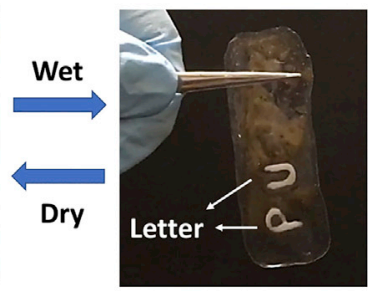

Figure 6. The dual anticounterfeiting hydrochromic film is designed by the combination of three layers

(A) Schematic illustration of dual anticounterfeiting hydrochromic film.

(B) Photo of dual anticounterfeiting hydrochromic film: switchable "cloud" patterns and "PU" letters between dry and wet states.

- KEY RESOURCES TABLE

- RESOURCE AVAILABILITY

Lead contact

- Materials availability

- Data and code availability

- METHOD DETAILS

O Synthesis of anionic PU water dispersion

O Preparation of PU/PEI hydrochromic film

- Preparation of PU/PMANa hydrochromic film

○ Preparation of hydrochromic film for dual anticounterfeiting

Characterization

\section{SUPPLEMENTAL INFORMATION}

Supplemental information can be found online at https://doi.org/10.1016/j.isci.2021.102652.

\section{ACKNOWLEDGMENTS}

This work is supported by the Natural Sciences and Engineering Research Council of Canada (NSERC, grants RGPIN-2019-04650 and RGPAS-2019-00115).

\section{AUTHOR CONTRIBUTIONS}

Conceptualization, design, methodology, and analysis, P.S. and B.Z.; experiments, P.S., M.S., and M.L.; manuscript writing, P.S. and B.Z.

\section{DECLARATION OF INTERESTS}

The authors declare no competing interests.

Received: February 27, 2021

Revised: April 26, 2021

Accepted: May 21, 2021

Published: June 25, 2021

\section{REFERENCES}

Bohren, C.F. (1983). Absorption and Scattering of Light by Small Particles, Absorption and Scattering of Light by Small Particles (John Wiley \& Sons). https://doi.org/10.1088/0031-9112/35/ 3/025.

Cai, G., Wang, J., Eh, A.L.S., Chen, J., Qian, K., Xiong, J., Thangavel, G., and Lee, P.S. (2018). Diphylleia grayi-inspired stretchable hydrochromics with large optical modulation in the visible-near-infrared region. ACS Appl.
Mater. Inter. 10, 37685-37693. https://doi.org/10 1021/acsami.8b12490.

Choi, J., Hua, M., Lee, S.Y., Jo, W., Lo, C.Y., Kim S.H., Kim, H.T., and He, X. (2020). Hydrocipher: bioinspired dynamic structural color-based cryptographic surface. Adv. Opt. Mater. 8, 1-8. https://doi.org/10.1002/adom.201901259.

Huang, G., Xia, Q., Huang, W., Tian, J., He, Z., Li, B.S., and Tang, B.Z. (2019a). Multiple anti- counterfeiting guarantees from a simple tetraphenylethylene derivative - high-contrasted and multi-state mechanochromism and photochromism. Angew. Chem. Int. Ed. 58, 17814-17819. https://doi.org/10.1002/anie. 201910530.

Huang, W., Xu, M., Liu, J., Wang, J., Zhu, Y., Liu, Jia, Rong, H., and Zhang, J. (2019b). Hydrophilic doped quantum dots "ink" and their inkjetprinted patterns for dual mode anticounterfeiting 
by reversible cation exchange mechanism. Adv Funct. Mater. 29, 1-6. https://doi.org/10.1002/ adfm.201808762.

Jacucci, G., Schertel, L., Zhang, Y., Yang, H., and Vignolini, S. (2020). Light management with natural materials: from whiteness to transparency. Adv. Mater. 2001215. https://doi.org/10.1002/ adma.202001215.

Kang, H., Lee, J.W., and Nam, Y. (2018). InkjetPrinted multiwavelength thermoplasmonic images for anticounterfeiting applications. ACS Appl. Mater. Inter. 10, 6764-6771. https://doi org/10.1021/acsami.7b19342.

Lei, Y., Dai, W., Guan, J., Guo, S., Ren, F., Zhou, Y., Shi, J., Tong, B., Cai, Z., Zheng, J., and Dong, Y. (2020). Wide-range color-tunable organic phosphorescence materials for printable and writable security inks. Angew. Chem. Int. Ed. 59, 16054-16060. https://doi.org/10.1002/anie. 202003585

Li, Z., and Yin, Y. (2019). Stimuli-responsive optical nanomaterials. Adv. Mater. 31, 1-33. https://doi. org/10.1002/adma.201807061.

Liu, P., Chang, W., Ju, L., Chu, L., Xie, Z., Chen, J., and Yang, J. (2019a). Bioinspired noniridescent structural color with hidden patterns for anticounterfeiting. ACS Appl. Nano Mater. 2, 5752-5760. https://doi.org/10.1021/acsanm. $9 \mathrm{~b} 01218$.

Liu, Y., Han, F., Li, F., Zhao, Y., Chen, M., Xu, Z., Zheng, X., Hu, H., Yao, J., Guo, T., et al. (2019b). Inkjet-printed unclonable quantum dot fluorescent anti-counterfeiting labels with artificial intelligence authentication. Nat. Commun. 10, 1-9. https://doi.org/10.1038/ s41467-019-10406-7.

Peng, C.Y., Hsu, C.W., Li, C.W., Wang, P.L., Jeng, C.C., Chang, C.C., and Wang, G.J. (2018). Flexible photonic crystal material for multiple anticounterfeiting applications. ACS Appl. Mater. Inter. 10, 9858-9864. https://doi.org/10.1021/ acsami.8b00292.

Rybin, M.V., Khanikaev, A.B., Inoue, M., Samusev, K.B., Steel, M.J., Yushin, G., and Limonov, M.F. (2009). Fano resonance between Mie and bragg scattering in photonic crystals. Phys. Rev. Lett. 103, 23901. https://doi.org/10.1103/PhysRevLett. 103.023901 .
Si, P., Jiang, F., Cheng, Q.S., Rivers, G., Xie, H., Kyaw, A.K.K., and Zhao, B. (2020a). Triple noncovalent dynamic interactions enabled a tough and rapid room temperature self-healing elastomer for next-generation soft antennas. J. Mater. Chem. A 8, 25073-25084. https://doi. org/10.1039/d0ta06613c

Si, P., Yu, L., and Zhao, B. (2020b). Poly methacrylic acid sodium salt (PMANa)/ Polyurethane (PU) latex-polyelectrolyte colloid systems enabling one-pot fabrication of nonperiodic structured mechanoresponsive smart windows. ACS Appl. Mater. Inter. 12 27607-27613. https://doi.org/10.1021/acsami. 0c06943.

Singh, V.K., Chitumalla, R.K., Ravi, S.K., Zhang, Y. Xi, Y., Sanjairaj, V., Zhang, C., Jang, J., and Tan, S.C. (2017). Inkjet-printable hydrochromic paper for encrypting information and anticounterfeiting. ACS Appl. Mater. Inter. 9, 33071-33079. https://doi.org/10.1021/acsami. $7 b 08054$.

Sun, J., and Bhushan, B. (2019)

Nanomanufacturing of bioinspired surfaces. Tribol. Int. 129, 67-74. https://doi.org/10.1016/j. triboint.2018.08.007.

Syurik, J., Siddique, R.H., Dollmann, A., Gomard G., Schneider, M., Worgull, M., Wiegand, G., and Hölscher, H. (2017). Bio-inspired, large scale, highlyscattering films for nanoparticlealternative white surfaces. Sci. Rep. 7, 1-11. https://doi.org/ 10.1038/srep46637.

Vigneron, J.P., Pasteels, J.M., Windsor, D.M. Vértesy, Z., Rassart, M., Seldrum, T., Dumont, J., Deparis, O., Lousse, V., Biró, L.P., et al. (2007). Switchable reflector in the Panamanian tortoise beetle Charidotella egregia (Chrysomelidae: cassidinae). Phys. Rev. E 76, 31907. https://doi. org/10.1103/PhysRevE.76.031907.

Vüllers, F., Gomard, G., Preinfalk, J.B., Klampaftis, E., Worgull, M., Richards, B., Hölscher, H., and Kavalenka, M.N. (2016). Bioinspired superhydrophobic highly transmissive films for optical applications. Small 12, 6144-6152. https:// doi.org/10.1002/smll.201601443.

Wang, Y.M., Tian, X.T., Zhang, H., Yang, Z.R., and Yin, X.B. (2018). Anticounterfeiting quick response code with emission color of invisible metal-organic frameworks as encoding information. ACS Appl. Mater. Inter. 10, 22445 22452. https://doi.org/10.1021/acsami.8b06901.

Xiao, L., Wu, Y., Yu, Z., Xu, Z., Li, J., Liu, Y., Yao, J., and $\mathrm{Fu}, \mathrm{H}$. (2018). Room-temperature phosphorescence in pure organic materials: halogen bonding switching effects. Chemistry 24, 1801-1805. https://doi.org/10.1002/chem. 201705391.

Yao, X., Bai, Y., Lee, Y.J., Qi, Z., Liu, X., and Yin, Y. (2019). Multi-colored hollow carbon-containing titania nanoshells for anti-counterfeiting applications. J. Mater. Chem. C 7, 14080-14087. https://doi.org/10.1039/c9tc04146j.

Yong, J., Chen, F., Yang, Q., Du, G., Shan, C., Bian, H., Farooq, U., and Hou, X. (2015). Bioinspired transparent underwater superoleophobic and anti-oil surfaces. J. Mater. Chem. A 3, 9379-9384. https://doi.org/10.1039/ c5ta01104c.

Yoo, G.Y., Lee, S.J., Ko, M., Kim, H., Lee, K.N., Kim, W., and Do, Y.R. (2020). Diphylleia grayiinspired intelligent hydrochromic adhesive film. ACS Appl. Mater. Inter. https://doi.org/10.1021/ acsami.0c13185.

Yu, X., Wu, L., Hu, H., Chen, M., Tan, Y., Yang, D., Pan, Q., Zhong, Q., Supasai, T., and Zhang, Q. (2018). Cs4PbX6 (X = Cl, Br, I) nanocrystals: preparation, water-triggered transformation behavior, and anti-counterfeiting application. Langmuir 34, 10363-10370. https://doi.org/10. 1021/acs.langmuir.8b01683.

Yu, X., Wu, L., Yang, D., Cao, M., Fan, X., Lin, H. Zhong, Q., Xu, Y., and Zhang, Q. (2020). Hydrochromic CsPbBr3 nanocrystals for anticounterfeiting. Angew. Chem. Int. Ed. 59, 1452714532. https://doi.org/10.1002/anie.202005120.

Zeng, S., Li, R., Freire, S.G., Garbellotto, V.M.M. Huang, E.Y., Smith, A.T., Hu, C., Tait, W.R.T., Bian, Z., Zheng, G., et al. (2017). Moisture-responsive wrinkling surfaces with tunable dynamics. Adv. Mater. 29, 1-7. https://doi.org/10.1002/adma. 201700828.

Zhao, H., Qin, X., Zhao, L., Dong, S., Gu, L., Sun, W., Wang, D., and Zheng, Y. (2020). Invisible inks for secrecy and anticounterfeiting: from single to double-encryption by hydrochromic molecules. ACS Appl. Mater. Inter. 12, 8952-8960. https:// doi.org/10.1021/acsami.0c00462. 


\section{STAR $\star$ METHODS}

\section{KEY RESOURCES TABLE}

\begin{tabular}{lll}
\hline REAGENT or RESOURCE & SOURCE & IDENTIFIER \\
\hline Chemicals, peptides, and recombinant proteins & & \\
\hline Polypropylene glycol-2000 (PPG-2000) & Sigma-Aldrich & CAT\#81380 \\
Dimethylolpropionic acid (DMPA) & Sigma-Aldrich & CAT\#106615 \\
Isophorone diisocyanate (IPDI) (98\%) & Sigma-Aldrich & CAT\#317624 \\
Ethylene diamine (EDA) (99.5\%) & Sigma-Aldrich & CAT\#391085 \\
Triethylamine (TEA) (99\%) & Sigma-Aldrich & CAT\#T0086 \\
Reagent acetone & Sigma-Aldrich & CAT\#179124 \\
Branched polyethylenimine (PEI) (average & Sigma-Aldrich & CAT\#408727 \\
Mw $\approx 25000)$ & & \\
Ammonium hydroxide solution (28-30\%) & Sigma-Aldrich & CAT\#221228 \\
Poly (methacrylic acid, sodium salt) solution & Sigma-Aldrich & CAT\#674044 \\
(PMANa, Mw $\approx 4000-6,000,40$ wt $\%$ in $\left.\mathrm{H}_{2} \mathrm{O}\right)$ & & \\
\hline
\end{tabular}

\section{RESOURCE AVAILABILITY}

\section{Lead contact}

Further information and requests for resources and reagents should be directed to and will be fulfilled by the lead contact, Boxin Zhao (zhaob@uwaterloo.ca).

\section{Materials availability}

This study did not generate new unique reagents.

\section{Data and code availability}

This study did not generate/analyze [datasets/code]

\section{METHOD DETAILS}

\section{Synthesis of anionic PU water dispersion}

$50 \mathrm{~g}$ of PPG-2000, $3.4 \mathrm{~g}$ of DMPA and $16 \mathrm{~mL}$ of IPDI were added into a three-neck flask equipped with a water-cooled condenser and $\mathrm{N}_{2}$ tubes. The reaction took place for $4 \mathrm{hr}$ at $110^{\circ} \mathrm{C}$ under mechanical stirring; the resulting polymer was cooled down to $40^{\circ} \mathrm{C}$, and then $38 \mathrm{~mL}$ of reagent acetone was added into the flask under mechanical stirring; then, $100 \mathrm{~mL}$ of amine water solution containing $0.018 \mathrm{~mol}$ EDA and 0.022 mol TEA was added into flask under vigorous stirring at room temperature. Acetone was removed by vacuum oven for $20 \mathrm{~min}$. Anionic PU water dispersion was obtained and condensed to $30 \mathrm{wt} \%$ solid content.

\section{Preparation of PU/PEI hydrochromic film}

$2 \mathrm{~mL}$ anionic PU water dispersion was cast on $75 \mathrm{~mm}$ by $25 \mathrm{~mm}$ glass slides. The transparent PU film was obtained after drying naturally at room temperature for $24 \mathrm{hr}$. Branched PEI was dissolved in DI water with a concentration of $30 \mathrm{mg} / \mathrm{mL}$. $0.3 \mathrm{~mL}$ ammonium hydroxide solution and $0.3 \mathrm{~mL}$ branched PEI water solution were added into $2 \mathrm{~mL}$ of the anionic PU water dispersion. The obtained PU-PEI solution was spray coated on transparent PU film via air brush and then dried at room temperature for $30 \mathrm{~min}$.

\section{Preparation of PU/PMANa hydrochromic film}

$2 \mathrm{~mL}$ anionic PU water dispersion was cast on $75 \mathrm{~mm}$ by $25 \mathrm{~mm}$ glass slides. The transparent PU film was obtained after drying naturally at room temperature for $24 \mathrm{hr}$. PMANa solution was diluted to $4 \mathrm{wt} \%$ and then $2 \mathrm{~mL}$ PMANa solution was mixed with $2 \mathrm{~mL}$ anionic PU water. $0.5 \mathrm{~mL}$ PU/PMANa solution was cast on PU film followed by drying at room temperature for $2 \mathrm{hr}$. 
Preparation of hydrochromic film for dual anticounterfeiting

$2 \mathrm{~mL}$ anionic PU water dispersion was cast on $75 \mathrm{~mm}$ by $25 \mathrm{~mm}$ glass slides. The transparent PU film was obtained after drying naturally at room temperature for $24 \mathrm{hr}$. PU/PEI solution was spray coated on PU film via a stencil with a "cloud" pattern to produce a thin layer "cloud" pattern of PU/PEI with around $5 \mu \mathrm{m}$ thickness. After drying for $30 \mathrm{~min}$ at room temperature, the film was peeled off from the glass slide and then flipped over to attach on the glass slide. The "PU" letters were handwritten via a glass pipette filled with PU/PMANa solution followed by drying at room temperature for $2 \mathrm{hr}$. The "cloud" pattern was visible in the air and changed to "PU" letters after soaking the obtained hydrochromic film in water for $3 \mathrm{~s}$. After drying, the "PU" letters changed back to the "cloud" pattern.

\section{Characterization}

Dynamic light scattering (DLS) (Malvern Zetasizer Nano ZSP) was used to measure the average diameter and zeta potential of the PU, PU/PEI and PU/PMANa colloidal dispersions.

UV-vis diffuse reflectance spectroscopy (UV-Vis) (Lambda 1050) was performed to measure the visible light $(400 \mathrm{~nm}-800 \mathrm{~nm})$ transmittance of PU/PEI and PU/PMANa films in the dry and wet states. Tensile tests were conducted with a universal mechanical tester (UMT)

(T1377, Center for Tribology Inc.) at a speed of $2 \mathrm{~mm} / \mathrm{s}$ using a $10 \mathrm{~kg}$ load cell. 180-degree peeling tests were completed with UMT at a speed of $0.5 \mathrm{~mm} / \mathrm{s}$ using a $1 \mathrm{~kg}$ load cell. A backing Scotch tape with $19 \mathrm{~mm}$ width was adhered on samples for peeling. Scratch tests were performed with UMT by using a $1 \mathrm{~mm}$ diameter stainless steel ball indenter that was fixed on a $10 \mathrm{~kg}$ load cell. The indenter was moved horizontally along the film surface for $20 \mathrm{~mm}$ at $0.5 \mathrm{~mm} / \mathrm{s}$ while the normal force linearly increased from $2 \mathrm{~g}$ to $5000 \mathrm{~g}$. A digital microscope (AD4113ZT, Dino-Lite) was used to observe the scratch track. A 3D laser microscope (OLS5000, Olympus) was applied to measure the thickness of PU/PEI and PU/PMANa coatings on PU film. The morphology of PU/PEI and PU/PMANa films was observed using a SEM (ZEISS Ultra) at $10 \mathrm{kV}$ accelerating voltage. $5 \mu \mathrm{L}$ of DI water was used for measuring the water contact angle of PU/PEI and PU/ PMANa films. 